\title{
Circuit
}

Musiques contemporaines

\section{Clés du Manuscrit trouvé à Saragosse Understanding the Manuscrit trouvé à Saragosse}

\section{José Evangelista}

Volume 12, numéro 2, 2002

Opéra aujourd'hui

URI : https://id.erudit.org/iderudit/902252ar

DOI : https://doi.org/10.7202/902252ar

Aller au sommaire du numéro

Éditeur(s)

Les Presses de l'Université de Montréal

ISSN

1183-1693 (imprimé)

1488-9692 (numérique)

Découvrir la revue

Citer cet article

Evangelista, J. (2002). Clés du Manuscrit trouvé à Saragosse. Circuit, 12(2),

53-68. https://doi.org/10.7202/902252ar

\section{Résumé de l'article}

L'adaptation du roman Manuscrit trouvé à Saragosse constituait un défi en raison de la complexité de la trame narrative et de la multitude de personnages. L'article expose comment la mise en musique participe à l'identification de chaque personnage à partir de références culturelles et sociales qui lui sont propres. Les différents personnages sont ensuite classés par groupes et quelques situations permettent aussi le recours à des styles musicaux référentiels. Le tout est illustré par une vingtaine d'exemples tirés de l'opéra. 


\title{
Clés du Manuscrit trouvé à Saragosse
}

\author{
José Evangelista
}

J'ai pris connaissance du Manuscrit trouvé à Saragosse de Jean Potocki à la fin des années soixante, dans la traduction espagnole de l'édition de Roger Caillois (Gallimard, 1958). Cela représentait à peu près un tiers du roman, qui n'a connu d'édition complète qu'en 1989 (Éditions José Corti). Au début des années quatrevingt-dix, j'ai parlé à Alexis Nouss de mon projet d'écrire un opéra à partir du Manuscrit, et ensemble, nous avons déterminé les lignes générales de l'adaptation pour la scène.

La densité et la complexité du roman représentaient un défi considérable. II fallait garder la structure des récits imbriqués verticalement par les mises en abîme et horizontalement par le croisement des personnages et des situations d'une histoire à une autre. Nous sommes arrivés à une sélection d'histoires où l'on retrouve les différents genres présents dans le Manuscrit (roman fantastique, roman picaresque, histoire de brigands, conte libertin, roman philosophique...) et à une trentaine de personnages qui sont incarnés par neuf chanteurs.

\section{La mise en musique}

Avec la mise en musique, je me suis proposé de mettre en évidence le caractère narratif de l'opéra et de rendre compréhensibles les divers niveaux de la trame.

Pour souligner la différence entre récit et discours, j'ai établi quelques conventions dont la plus importante est que la narration est récitée alors que les dialogues sont chantés. Je me suis basé sur l'expérience acquise dans certaines de mes œuvres précédentes qui appartenaient au genre monodrame (Plume, texte d'H. Michaux; La Porte, texte d'A. Nouss, à partir de Kafka; Bukowski Stories, 
texte de Ch. Bukowski ; Alice \& Friends, texte de L. Carroll). Dans ces pièces, la voix soliste incarne tous les personnages de l'histoire, y compris celui du narrateur.

On se perd facilement dans le jeu de miroirs des histoires du roman, et ce, malgré le temps et l'espace limités (deux mois en Andalousie). Ainsi, à plusieurs reprises, les personnages se déclarent désorientés dans la multiplicité des récits enchevêtrés. Le géomètre dit : "Je ne sais vraiement qui parle et qui écoute. Tantôt le marquis de Val Florida raconte ses aventures à sa fille, tantôt celle-ci les rapporte au chef bohémien, qui à son tour nous les repète. C'est un vrai labyrinte» $128^{\mathrm{e}}$ journée). Pour rendre compréhensibles les niveaux de la trame, je me suis proposé d'identifier musicalement les personnages et de refléter la diversité de genres présents dans le roman. Par conséquent, j'emploie une variété de styles musicaux associés à des personnages identifiés par leur origine religieuse, culturelle ou sociale, ou encore identifiés par leur caractère; et à des situations (le gibet, les déplacements, les ébats amoureux, etc.).

\section{Styles associés à des personnages}

Le protagoniste, Alphonse van Worden, est le fil conducteur de l'histoire. II chante une musique partiellement atonale sur un fond $d$ 'accords par quintes. Le chanteur qui interprète Alphonse est le seul qui n'incarne qu'un seul personnage.

Extrait 1 : Alphonse

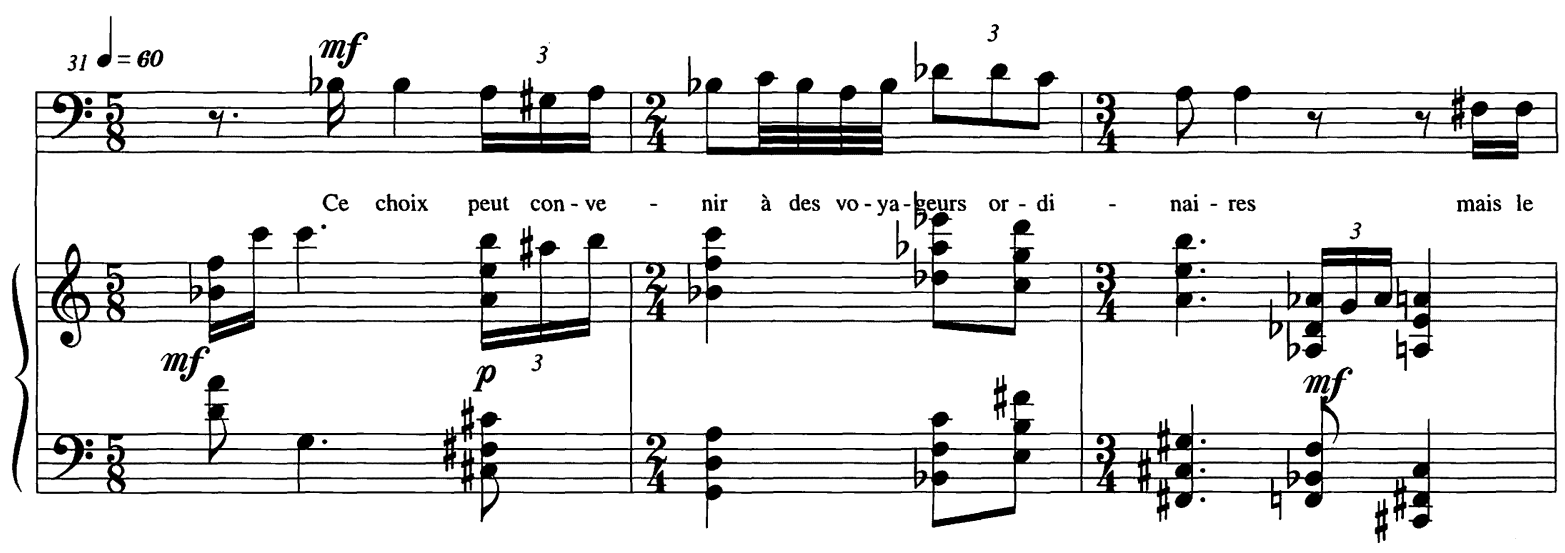


La majorité des autres personnages peut être présentée en quatre groupes: espagnols, mauresques, juifs et chrétiens :

\section{A) Les personnages espagnols}

a) personnages en rapport avec le monde andalou et gitan

- Mosquito, le valet d'Alphonse, est accompagné par une guitare dont le jeu en rasgueado est doublé par les cordes.

- Les deux bohémiennes : les tournures mélodiques ainsi que l'accompagnement sont librement inspirés du flamenco, tel que stylisé par Falla et Turina. Un emprunt plus direct au flamenco est le chant de la copla Cuando mi Paco... dans la scène 10. Les paroles, tirées du roman, semblent être une citation d'un poème populaire. La musique imite les bulerías mais a été composée par moi.

Extrait 2 : deux gitanes

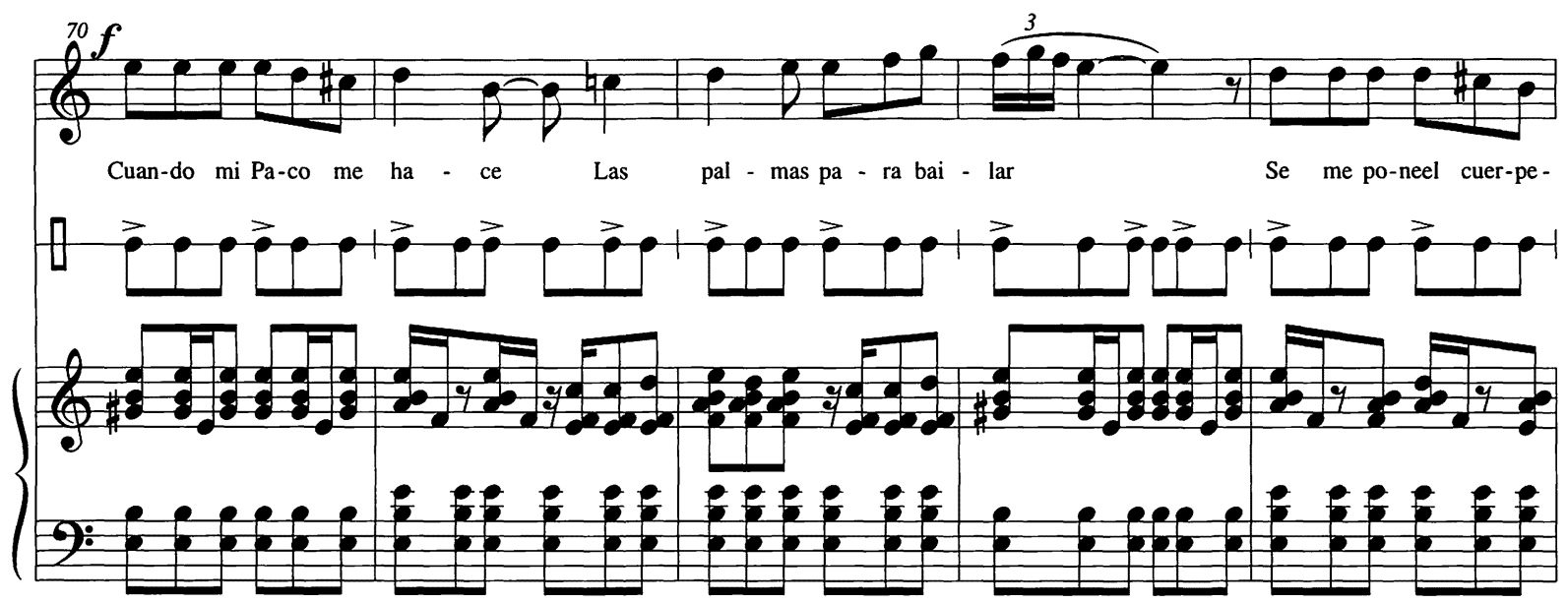


- Avadoro, le chef bohémien, chante en style andalou dans les dialogues et ses interventions sont précédées d'un court motif extrait du genre flamenco soleá. Pour ses récits, il emprunte le style du romance castillan, poème traditionnel à caractère monotone et répétitif qui sert à raconter des histoires. Avadoro chante une mélodie, El robo del Sacramento, de la région de Palencia.

\section{Extrait 3 : Avadoro}

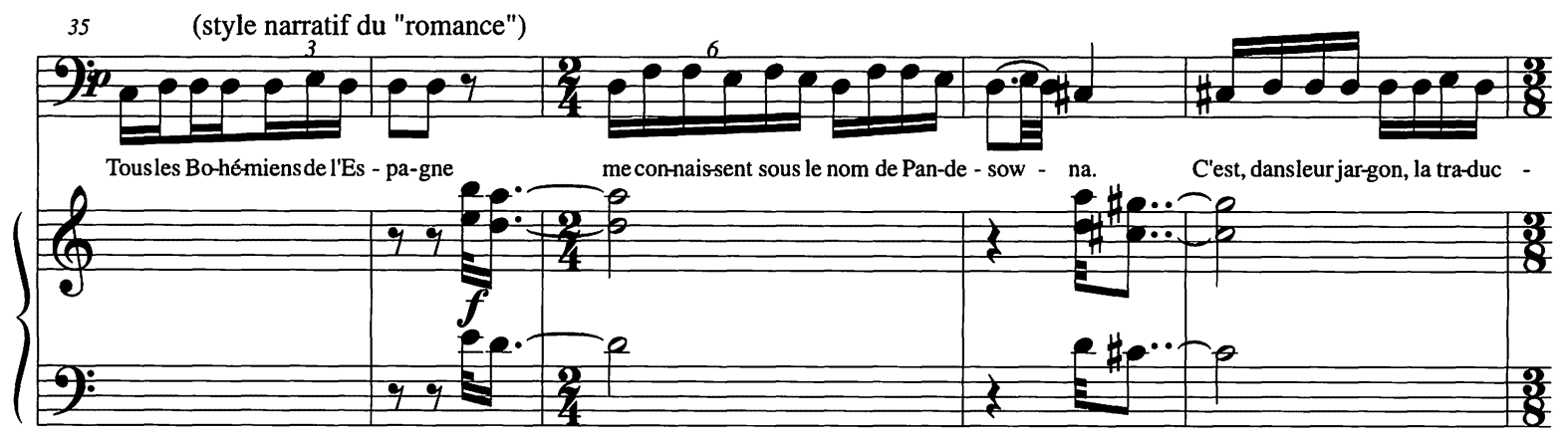

- Camille et Inésille chantent des mélodies inspirées du folklore d'autres régions : par exemple, l'air de Camille Tu as tort de t'affliger est une adaptation de El Niño perdido d'Estrémadoure.

Extrait 4 : Camille

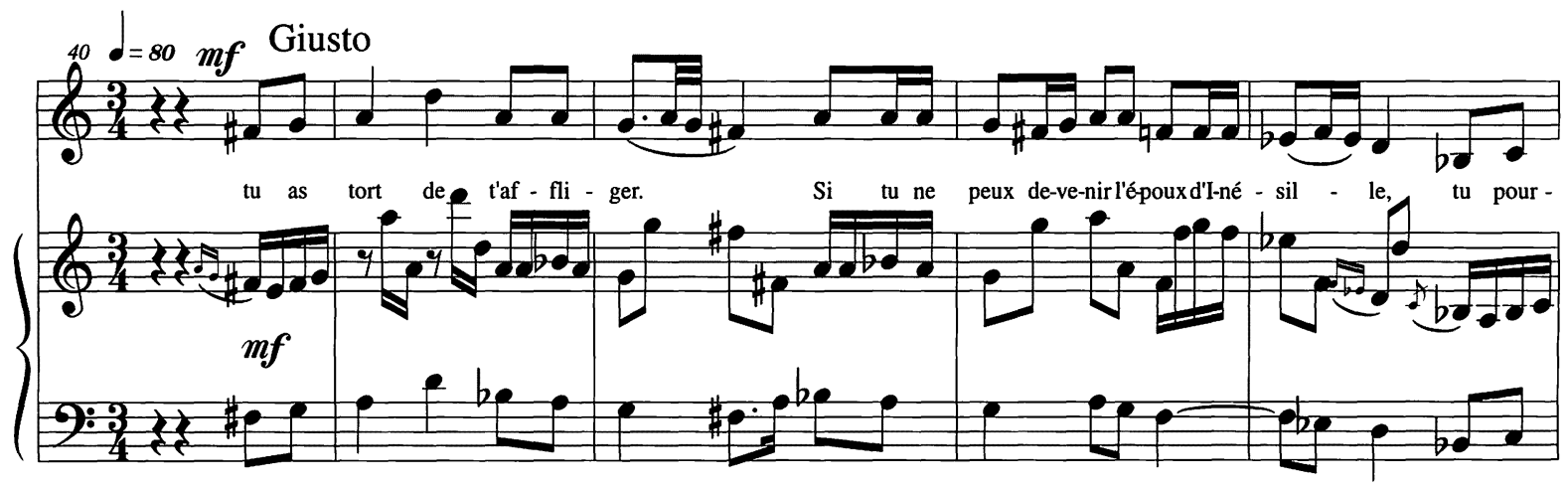


b) personnages espagnols à caractère noble

- Au chevalier de Tolède est associé le boléro, danse semi-populaire qui convient à son caractère citadin. Le traitement de la mélodie est monodique avec ornementation. C'est cette même technique que j'ai amplement utilisée dans d'autres œuvres (Monodías Españolas, Airs d'Espagne).

- Busqueros chante le romance la honra robada (province de Soria), lorsqu'il commence à raconter son histoire.

Extrait 5 : Busqueros

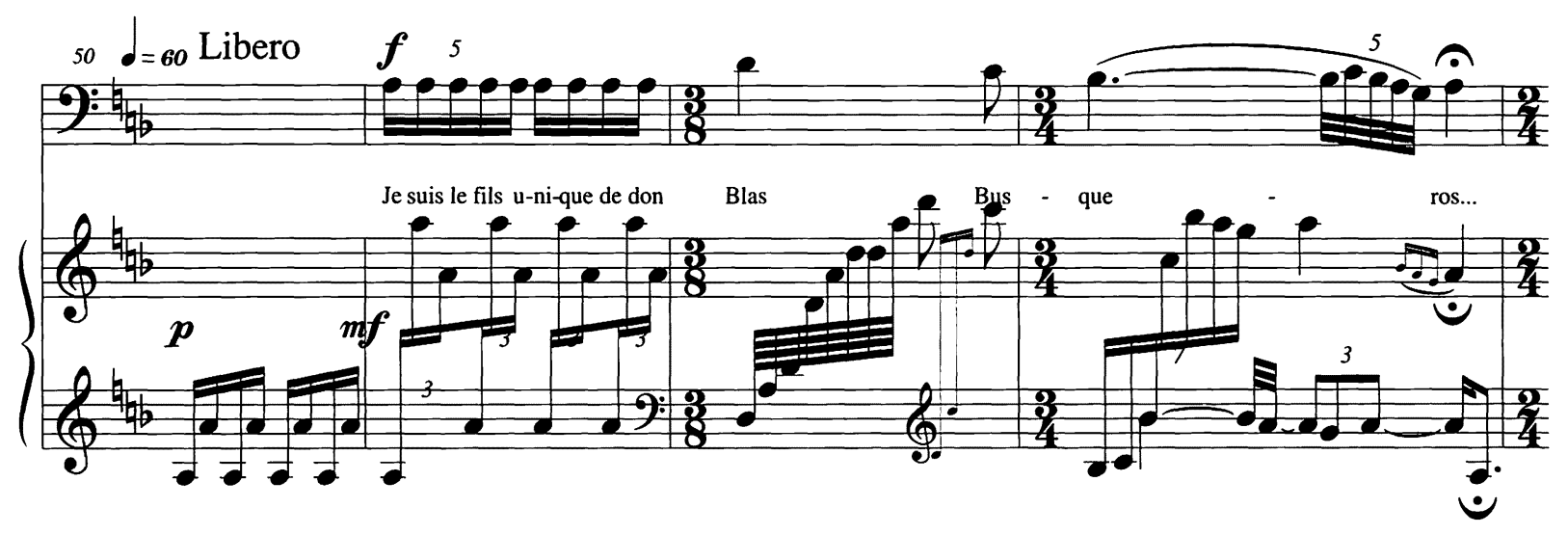

- Le capitaine espagnol fait une courte intervention basée sur le romance de Don Boyso (Asturies).

\section{B) Les personnages mauresques}

- Les deux cousines, Emina et Zibbedé, chantent des mélodies en style libre ou mesuré sur un accompagnement hétérophone, en superposant beaucoup d'ornementation à la mélodie. Les chants s'inspirent librement de la musique improvisée de l'Iran, l'Iraq et l'Égypte. 
Extrait 6 : Emina et Zibbedé

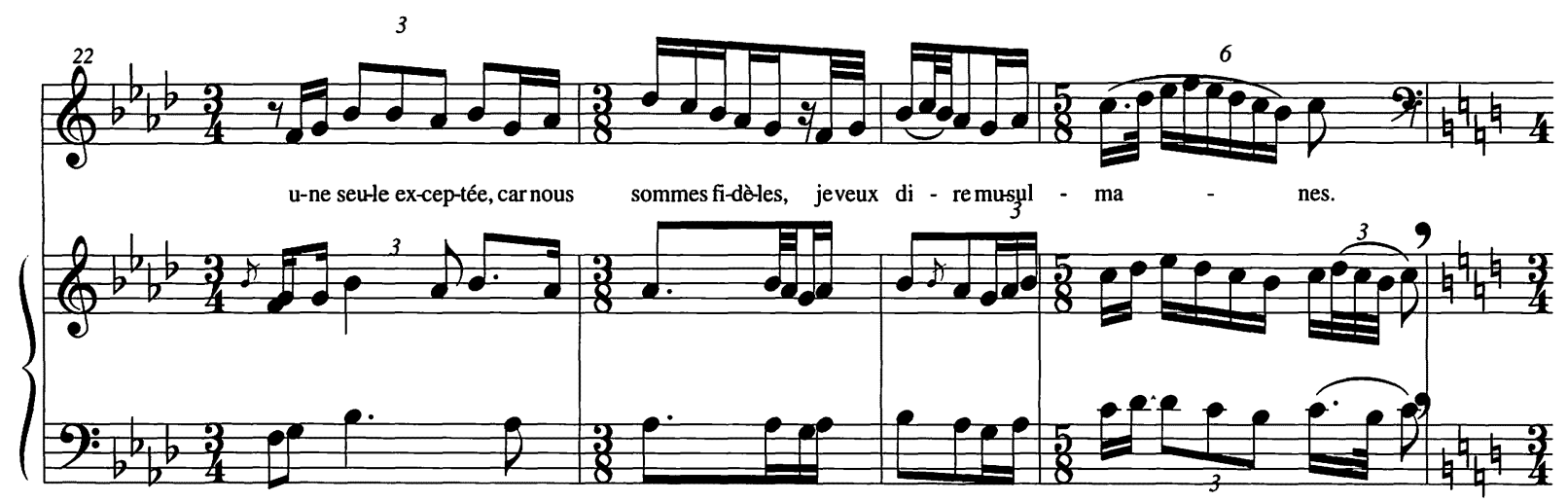

- Le scheik fait un long solo à la scène 23, celui-ci est conçu comme une improvisation iranienne : la ligne mélodique évolue à partir de motifs typés par les intervalles et par le dessin mélodique. Aussi, dans les musiques classiques du monde arabe, le passage d'un motif à un autre ressemble à une modulation, car il y a changement du matériau modal. Le solo du scheik est très modulant, changeant souvent d'armure et de tonalité. Mais, à la différence des musiques traditionnelles de l'Islam, j'emploie des motifs inventés qui évoluent dans le système chromatique des 12 sons.

Extrait 7 : le scheik

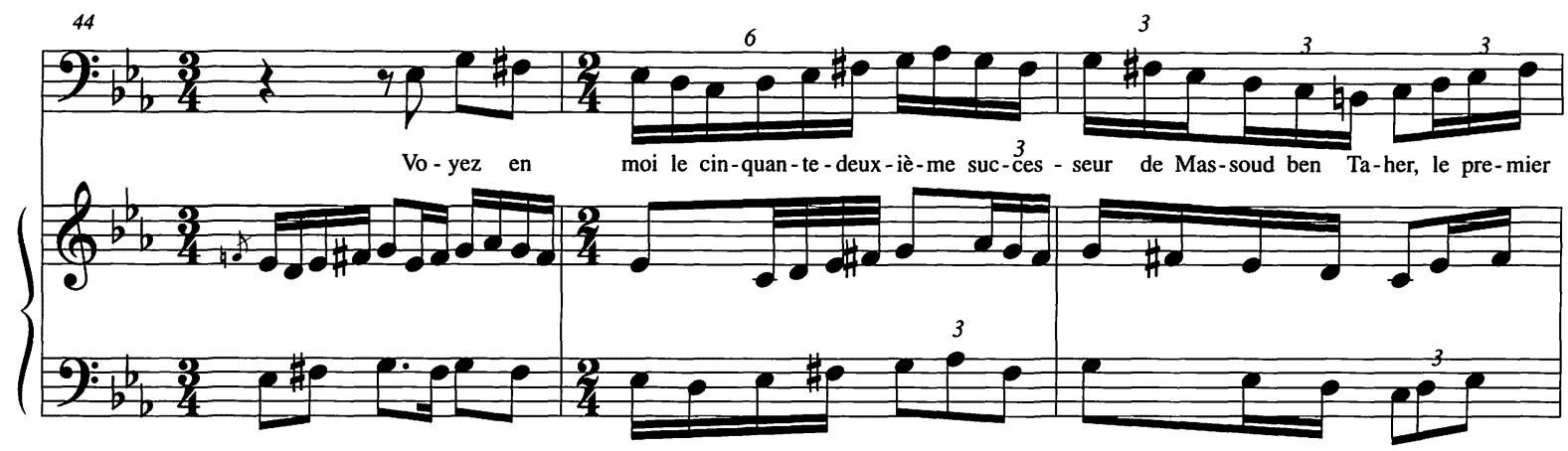

- Le derviche chante une adaptation libre de l'appel à la prière du muezzin. J'ai surtout retenu le motif rythmique de fin de phrase $0 . \lambda_{9}$ 


\section{C) Les personnages juifs}

- Uzeda : chant librement inspiré de la musique liturgique juive avec une citation de la lecture des te'amim (cantilation biblique) du rite ashkénaze. II est accompagné par le shofar, corne de bélier, seul instrument employé dans la liturgie juive. Le shofar est appuyé par d'autres instruments de l'orchestre afin de rendre contrôlable son émission sonore souvent imprévisible.

\section{Extrait 8 : Uzeda}

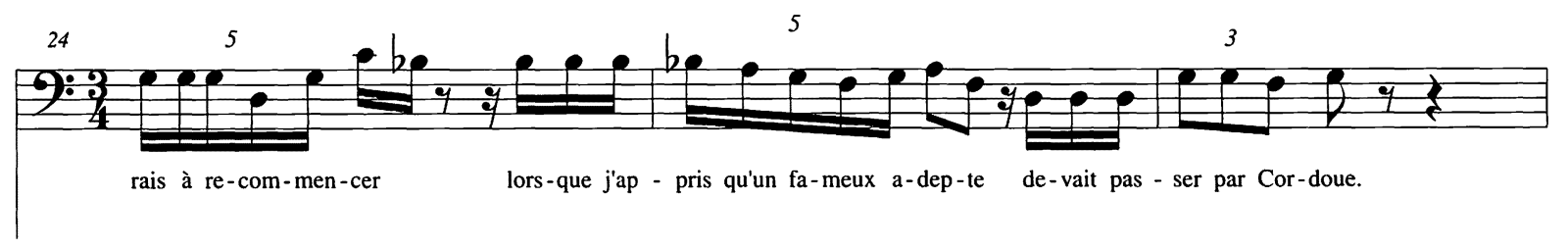

- Le petit rabbin bleu chante une adaptation d'une prière du Sabbath du Caucase.

- L'ange chante une harmonisation d'une cantilation biblique faisant partie d'un bar mitzvah (cérémonie d'initiation des garçons), de tradition judéoportugaise.

- Rébecca fait essentiellement deux types d'interventions. La première, plus fréquente et employée pour les dialogues et les narrations, est l'adaptation libre d'un chant liturgique sépharade de Casablanca. 
Extrait 9 : Rébecca

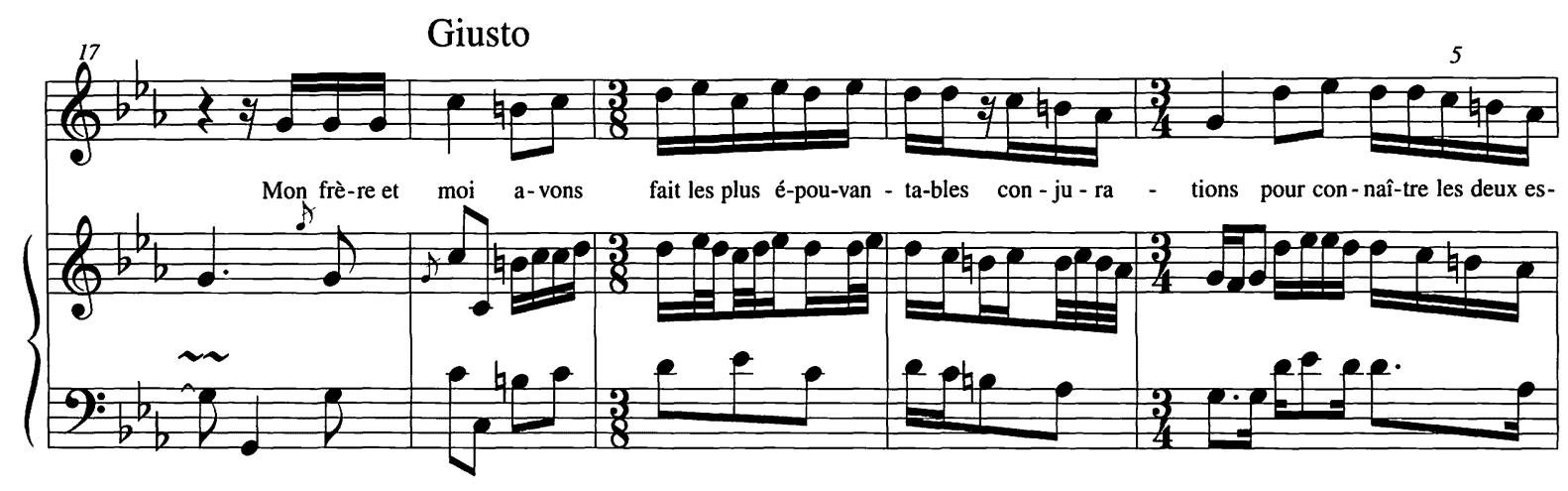

La deuxième, qui correspond aux passages lyriques, est de mon invention dans un style sans référence à la musique juive. Ce matériau réapparaît dans d'autres passages de l'opéra où l'on parle d'amour, par exemple, dans les interventions des cousines à la scène 7 .

\section{D) Les personnages de l'Église chrétienne}

- L'ermite chante un pastiche très libre de chant grégorien.

- L'inquisiteur emploie d'abord une ligne mélodique lente, vaguement inspirée du chant ecclésiastique. Ensuite, il adopte un style parlé pour décrire les supplices.

À ces quatre groupes s'en ajoute un cinquième, constitué de personnages identifiés par leur caractère :

- Pacheco : son récit s'appuie sur une ligne legato, chromatique et descendante.

- Le père de Pacheco : son intervention est basée sur une cellule rythmique.

- La femme de l'aubergiste : brève intervention marquée par des notes répétées staccato et par le tempo.

- Le père d'Alphonse : la ligne mélodique et l'accompagnement sont marqués par des intervalles de 5te augmentée et par une cellule rythmique. 
- Les Gémeaux célestes s'expriment en différents styles d'après les indications du roman (voir plus bas).

- Le géomètre chante dans un style staccato, en rythme binaire et dans un tempo rapide.

Extrait 10 : le géomètre

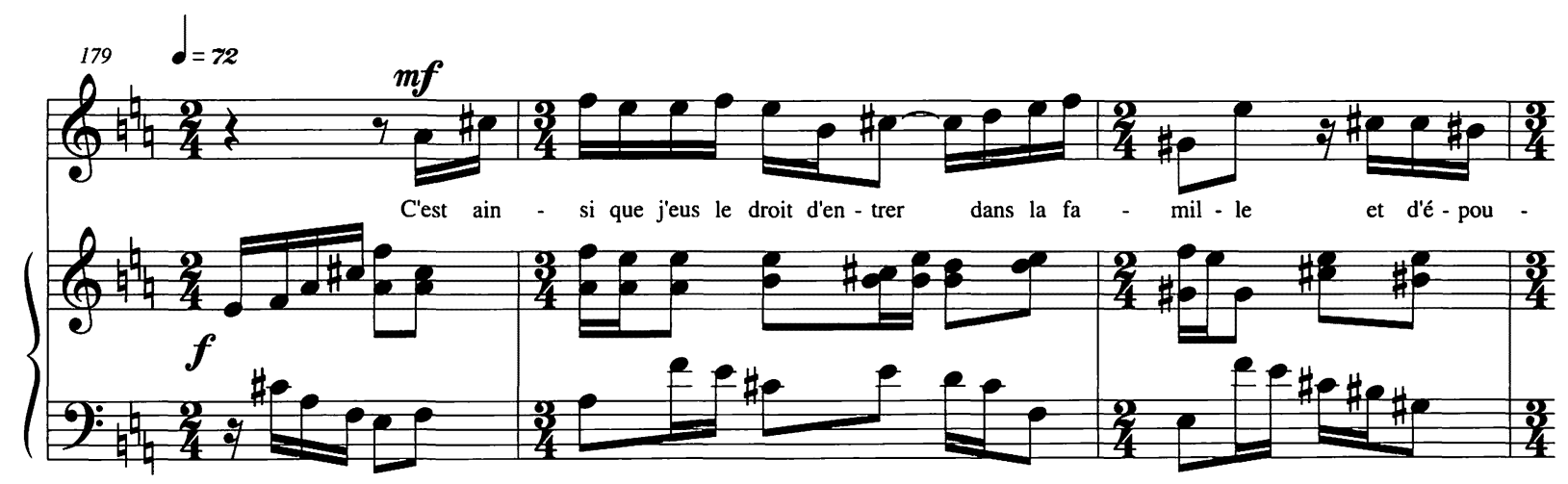

- Aguilar est accompagné d'une harmonie à quatre voix dans le registre moyen/grave. Ce sont des accords parfaits dans des positions souvent faibles et avec des enchaînements basés sur la fausse relation.

- Lope : sa ligne mélodique est souvent déduite de l'accompagnement instrumental.

- Busqueros : un style staccato en rythme binaire et une ligne mélodique à caractère pentatonique.

- Inés : style saccadé basé sur l'alternance de groupes de 3, 4 et 5 doubles croches. Dans la scène où Lope et Inés lisent les lettres qu'ils se sont écrites, chacun chante en empruntant le style de l'autre.

- L'officier français: je lui ai assigné un style atonal contemporain, qui se veut neutre dans le contexte de l'opéra. 
Les changements stylistiques se succèdent à l'intérieur d'un même dialogue, car chaque personnage donne la réplique dans le style qui lui est propre. Par exemple :

Extrait 11 : Emina, Alphonse, Emina
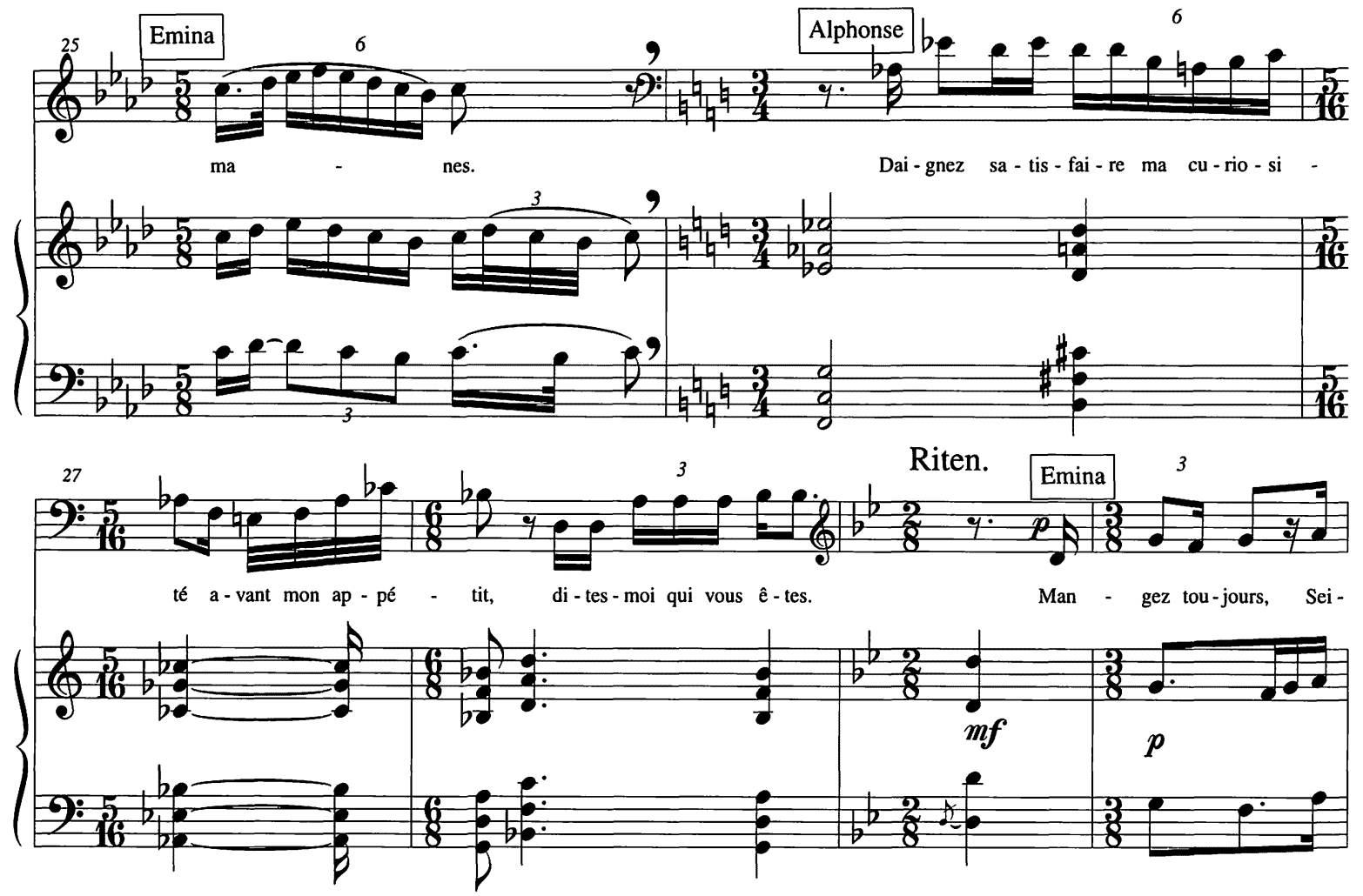

\section{Styles associés à des situations}

D'abord, j'ai suivi à la lettre les indications du roman chaque fois qu'il faisait référence à une pièce spécifique de musique. Par exemple, lors des échanges entre Rébecca et les Gémeaux célestes :

[Rébecca] y arrive alors que minuit sonne. Y pénètre, attirée et charmée par le chant de deux voix masculines, soutenues d'un instrument à cordes. 
Les Gémeaux exécutent une vocalise accompagnée par l'alto :

Extrait 12: deux Gémeaux

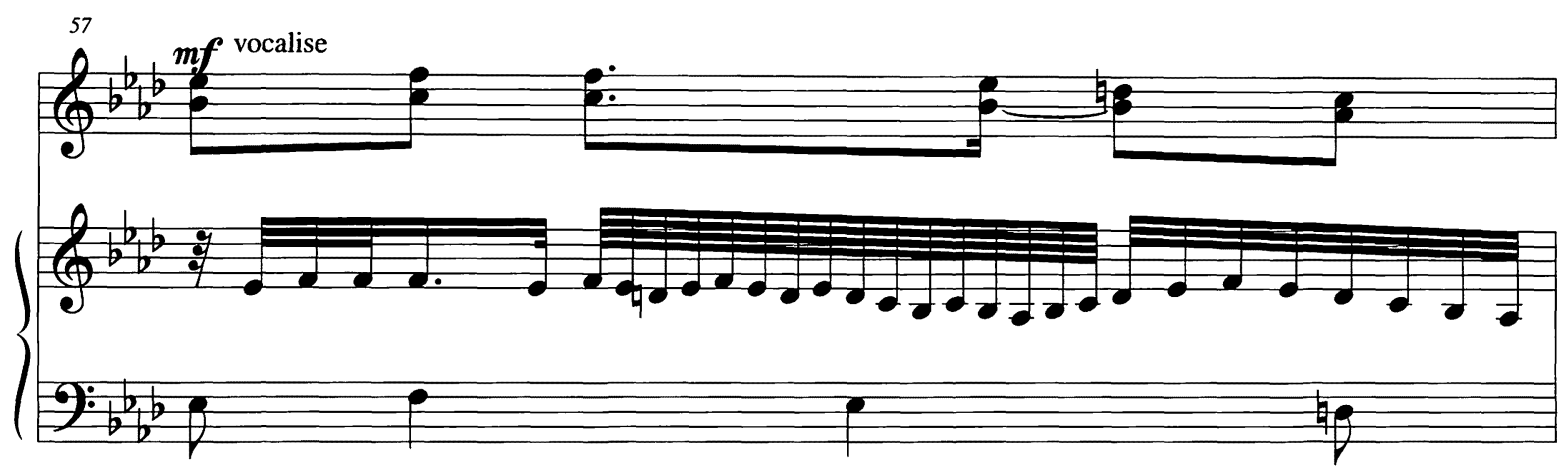

Elle prend du vin et se met à chanter une séguedille. Ils répondent "sur les mêmes rimes et la même pensée "...

Rébecca chante une séguedille manchega :

Extrait 13: Rébecca

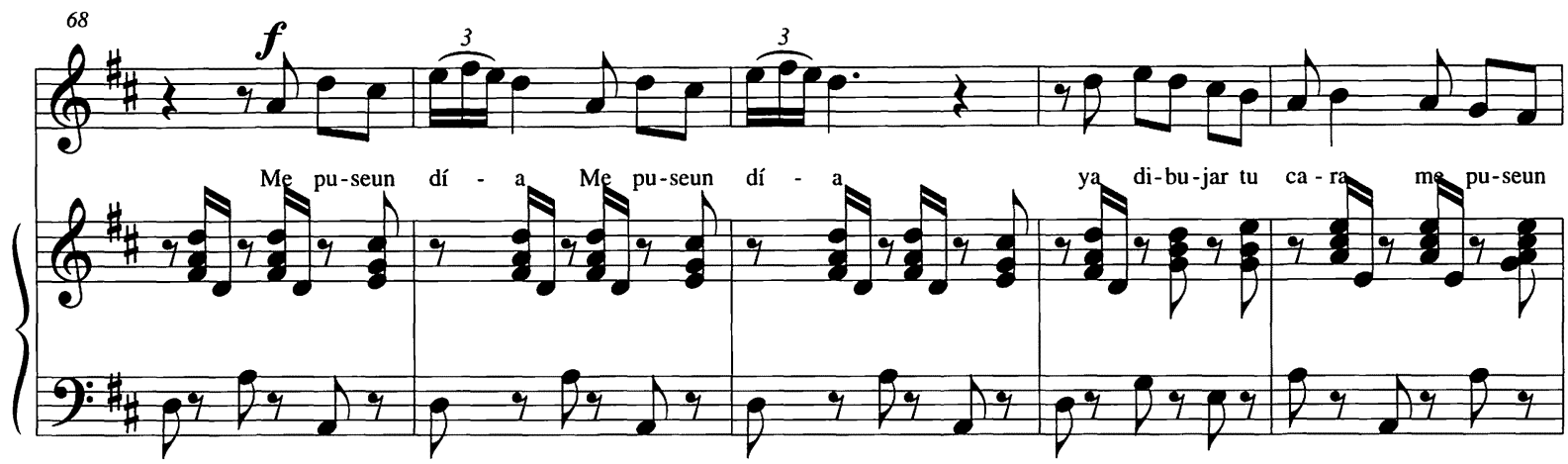


Enfin, lorsque les Gémeaux interprètent des chansons de leur pays, la Grèce, je cite deux passages d'un chant cleftique d'Épire : le premier est accompagné par le violon et la clarinette sur un bourdon de ud en trémolo:

Extrait 14 : premier Gémeau

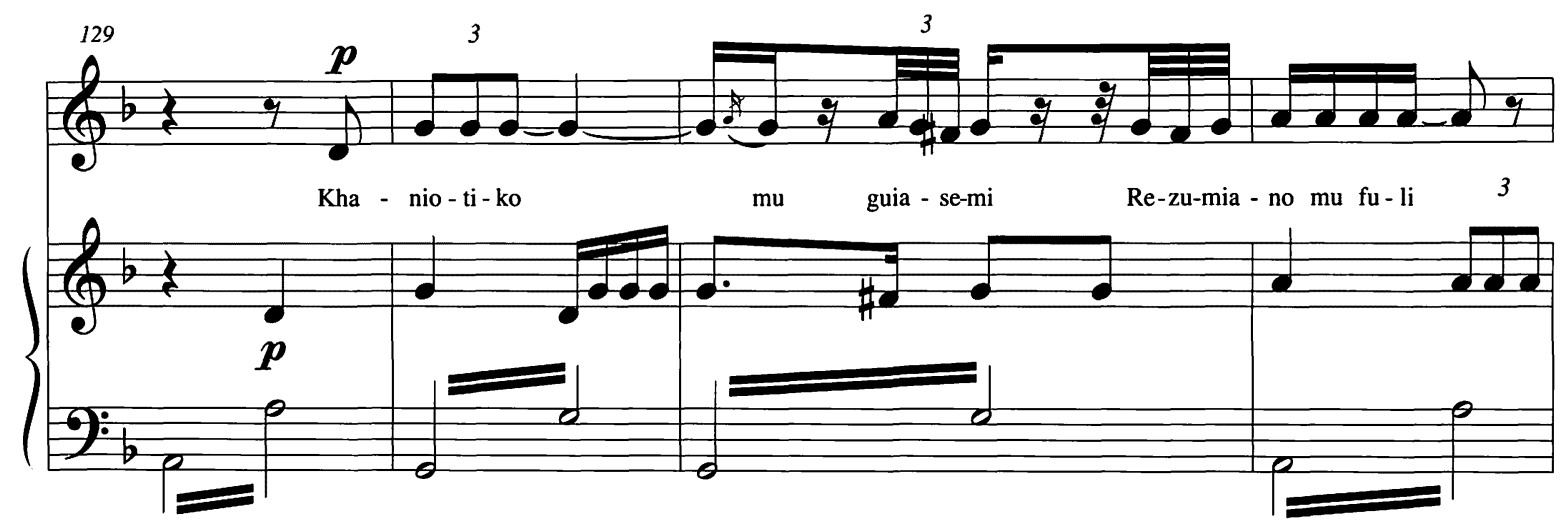

Vient ensuite une courte danse instrumentale pour clarinette et cordes (bouzouk plus pizz.).

\section{Extrait 15}

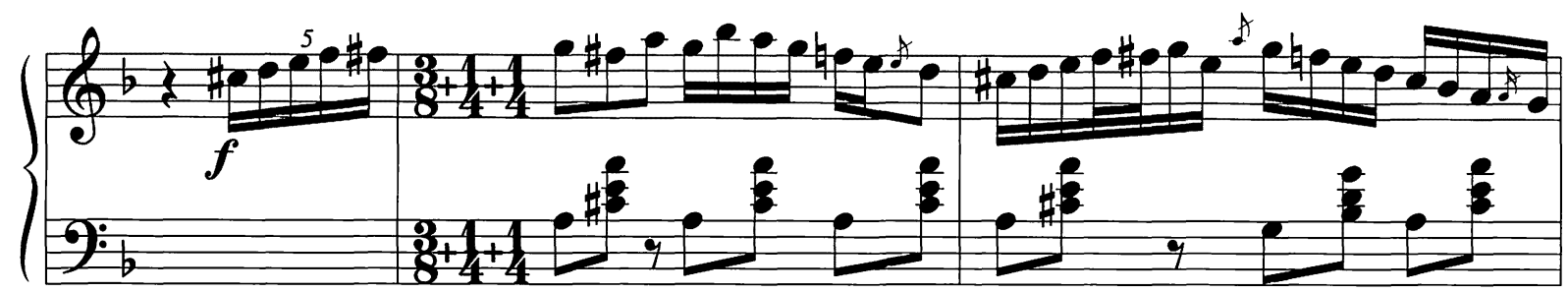

En plus de suivre les indications musicales du roman, j'ai associé des styles aux divers thèmes et situations :

- Les scènes mauresques, avec les deux cousines, le scheik, le derviche, etc. La danse d'Emina et Zibbedé est inspirée d'un rythme arabe à 10 temps (samai) mais la mélodie est inventée. Plusieurs scènes mauresques s'inspirent de la musique instrumentale arabo-andalouse du Maroc, la nûba. Par 
exemple, les débuts des scènes 2, 7 et 23 ont été composés dans l'esprit des préludes improvisés (tûshiya) de la nûba.

\section{Extrait 16}

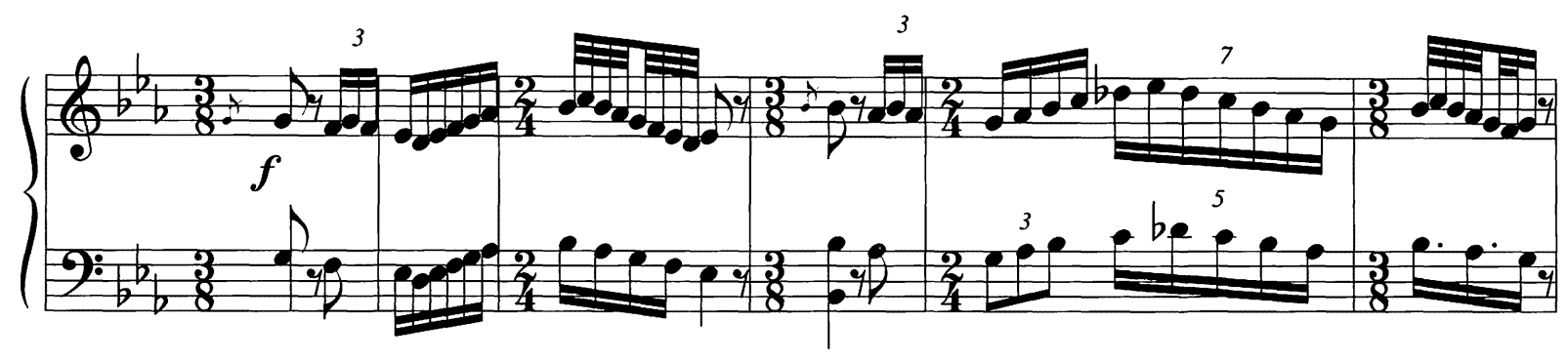

- Les scènes juives, chrétiennes et flamenco recréent des contextes appropriés aux personnages qui s'y rattachent.

- Le folklore traditionnel espagnol : Mosquito, Camille et Inésille chantent des mélodies populaires ou des imitations que j'ai créées. La séguedille, chantée par Rébecca et les Gémeaux, vient de la seguidilla manchega, la variante la plus représentative de ce chant. Les paroles sont traditionnelles, de même que le style musical, mais la mélodie est originale. Ce n'est pas du folklore imaginaire mais du folklore réel, bien que composé par moi, ce qui explique l'absence d'abstraction et de distance. Le chevalier de Tolède chante un boléro, mais sa réalisation n'est pas harmonique. Avadoro ef d'autres personnages chantent des romances.

- Le pastiche du XVIII siècle, employé lors de la scène du duel entre le père d'Alphonse et le marquis d'Urfé, est un menuet que j'ai écrit en m'inspirant librement du Père Soler.

\section{Extrait 17}

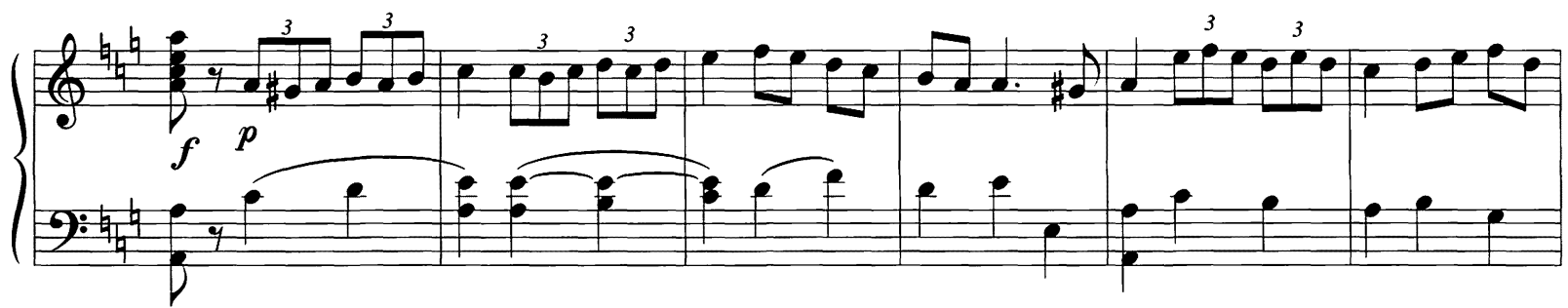


- Le pastiche de musique espagnole du Xx siècle, pour l'histoire de Lope Soarez, est une évocation qui constitue un pastiche au troisième degré. Cette musique est librement inspirée du compositeur espagnol Ernesto Halffter (1905-89), disciple de Manuel de Falla. Ses modèles sont Falla, naturellement, et Stravinsky, surtout celui de Pulcinella. Tout comme Stravinsky proposait le retour à Bach, Falla a montré le chemin aux jeunes compositeurs espagnols en proposant une relecture de Domenico Scarlatti, lui-même un Napolitain de naissance mais ayant composé la plupart de ses 555 sonates en Espagne. Voici comment j'interprète cette filiation : Scarlatti emprunte beaucoup de rythmes et de sonorités à la musique populaire espagnole de son époque; Falla imite le style de Scarlatti dans le Concerto pour clavecin; Ernesto Halffter crée un style personnel en s'appuyant sur le Concerto de Falla et sur Pulcinella. Enfin, je fais une parodie de cette musique. Un indice de l'emprunt est l'emploi du clavecin et d'harmonies diatoniques, bien que dissonantes, à la Stravinsky.

\section{Extrait 18}

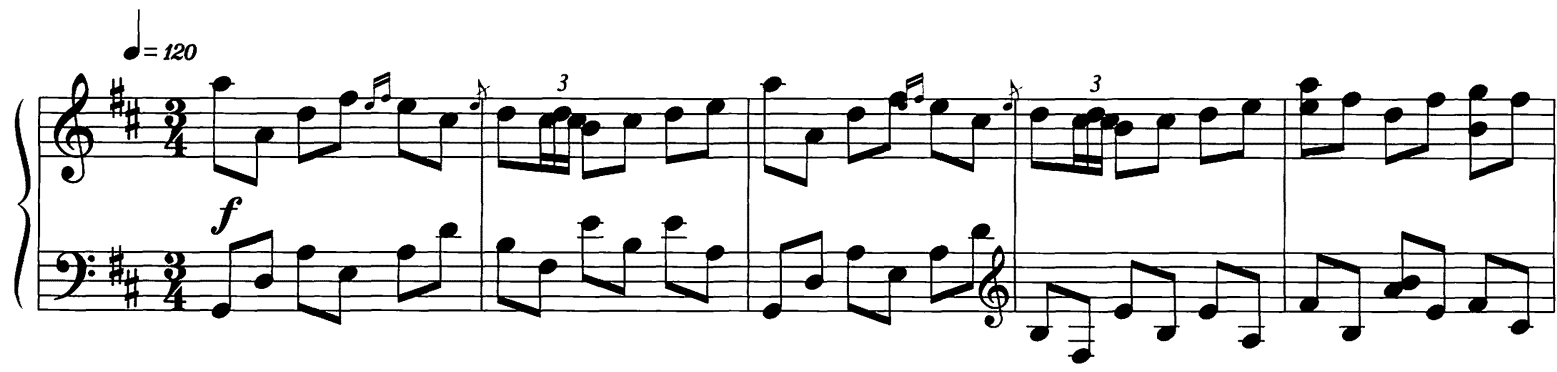

- Le premier passage instrumental de l'opéra est une sorte de thème du Manuscrit. II revient à plusieurs reprises, surtout dans la dernière scène et avant l'épilogue :

\section{Extrait 19}

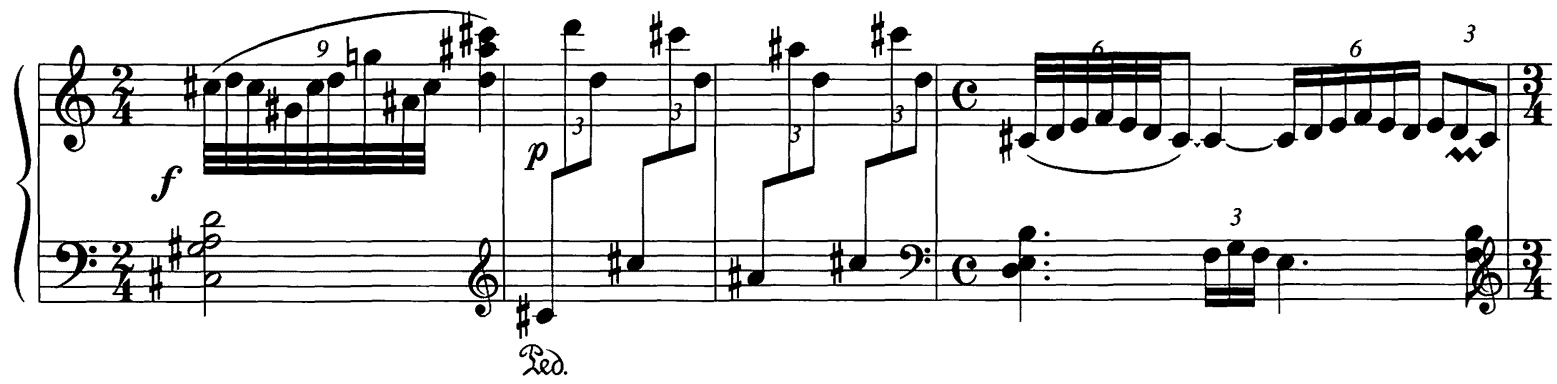


- Le gibet. Plusieurs personnages se réveillent sous le gibet de Los Hermanos, un lieu privilégié du roman (scènes $2,4,8,12$ et 13 ).

Extrait 20 : Alphonse

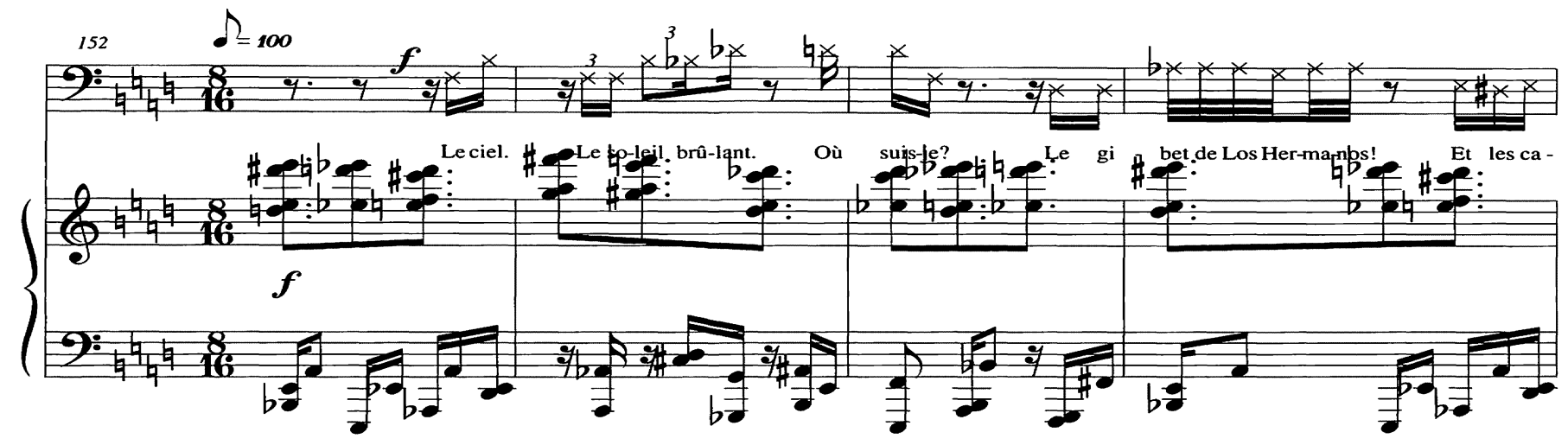

- Les ébats amoureux sont accompagnés par des musiques qui ont en commun le caractère harmonique et un type de construction polyphonique basé sur des motifs en forme d'arpèges répétés. Cela évoque l'abandon, la suspension du temps (scènes 4 et 7 ).

- Le thème du déplacement. Dans plusieurs scènes $(5,8,9$ et 22) la musique appuie le déplacement des personnages vers d'autres lieux.

\section{Extrait 21}

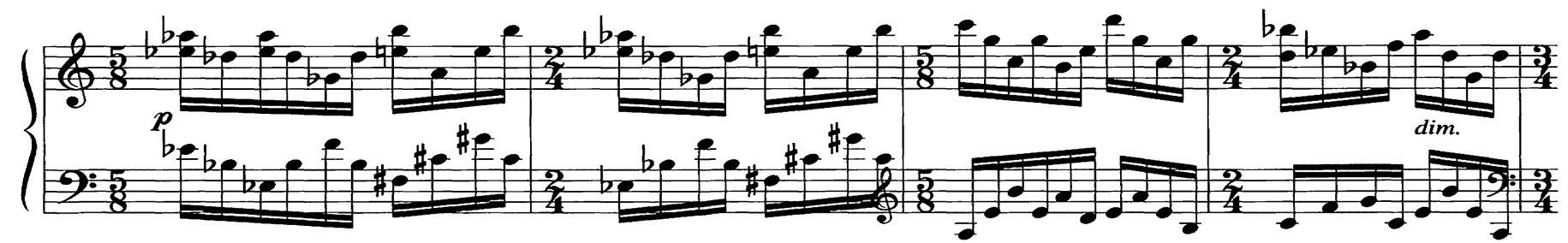

- Les références à la mine d'or et au trésor qu'Alphonse doit découvrir s'accompagnent d'accords de quintes superposées, évocation d'un son métallique! 
- Dans la scène finale, un court motif de trois notes accompagne l'apparition successive des personnages.

- Il y a deux références à des opéras célèbres : l'accompagnement sombre et grave d'Aguilar veut recréer l'esprit du Caronte de l'Orfeo de Monteverdi. Aussi, les trois coups au volet dans I'histoire d'Aguilar/Tolède et dans celle de Lope/Busqueros sont une référence aux coups du Commendatore dans Don Giovanni.

\section{Instrumentation}

C'est un petit orchestre de chambre : flûte, hautbois, clarinette, clavier électronique, percussion et quintette à cordes. La percussion emploie des hauteurs définies (vibraphone) et des instruments à hauteur non définie. Le clarinettiste doit jover aussi du shofar.

Le choix d'un clavier à la place d'un piano m'a permis de mieux appuyer l'ambiance reliée aux différentes scènes et aux personnages. Par exemple, je spécifie :

- son de guitare pour accompagner Mosquito, la danse bulerías et, en général, les scènes flamenco;

- son de qanun, cithare arabe, et son de ud, luth arabe, pour les scènes mauresques, spécialement avec les cousines et le scheik;

- son de bouzouk pour les chansons grecques;

- son de clavecin pour le duel (pastiche du XVIII siècle) et l'histoire de Lope Soarez;

- son de trompette pour renforcer le shofar.

Autrement, le clavier recourt à la harpe comme instrument en soi. Aussi, il double les cordes avec un son semblable lorsqu'il s'agit de textures en sons soutenus (par exemple, dans l'histoire d'Alphonse) et emploie d'autres timbres d'appui.

L'intrigue du Manuscrit est basée sur une mystification. L'ambiguitié entre réalité et apparence est présente partout. Dans la musique, à l'instar du roman, les choses ne sont pas toujours ce qu'elles semblent être : emprunt, pastiche et création s'entremêlent et se confondent. 